\title{
COEFICIENTES DE SENSIBILIDADE AO DÉFICIT HÍDRICO PARA A CULTURA DA BATATA NAS CONDIÇÕES EDAFOCLIMÁTICAS DA REGIÃO DE PIRACICABA, SP
}

\author{
Francisco Marcus Lima Bezerra ${ }^{1}$, Luiz Roberto Angelocci ${ }^{2}$ \& Keigo Minami ${ }^{3}$
}

\begin{abstract}
RESUMO
Este trabalho foi desenvolvido com o objetivo de se determinar o fator de sensibilidade da cultura da batata ao déficit hídrico $\left(\mathrm{k}_{\mathrm{y}}\right)$ em estádios isolados e no ciclo total da cultura, usando-se o modelo sugerido por Doorenbos e Kassam. O experimento foi conduzido em um solo Terra Roxa estruturada Latossólico, localizado no Campus da ESALQ, Piracicaba, SP, Brasil. O delineamento experimental adotado foi o de blocos ao acaso, com quatro repetições e oito tratamentos, definidos em função da época de indução do déficit hídrico nos três estádios fenológicos da batata, correspondendo à ocorrência de déficit hídrico em um, dois ou três estádios. O déficit correspondeu à aplicação da metade da lâmina média d'água do tratamento sem déficit hídrico e os resultados indicaram que o coeficiente de sensibilidade da batata, em cada estádio, variou na seguinte ordem decrescente: tuberização, enchimento de tubérculos e vegetativo, para déficit em apenas um estádio fenológico. Para a condição de déficit em dois estádios, os valores de $\mathrm{k}_{\mathrm{y}}$ foram todos superiores a 1 , tendo alcançado o maior valor na situação de déficits nos estádios tuberização e enchimento de tubérculos.
\end{abstract}

Palavras-chave: Solanum tuberosum, coeficiente de sensibilidade, estádios fenológicos

\section{SOIL WATER DEFICIT SENSIBILITY COEFFICIENTS FOR POTATO FOR EDAPHOCLIMATIC CONDITIONS OF PIRACICABA REGION, SP, BRAZIL}

\begin{abstract}
The objective of this research was to determine sensibility coefficients for potato under water deficit (ky) for isolated growing stages and for the whole lifecycle of the plant, using the model suggested by Doorenbos \& Kassam. The experiment was conducted in an area of Red structured Latossolic soil, at the ESALQ Campus, Piracicaba, SP, Brazil. The experimental design consisted of randomized blocks, with eight treatments and four replications, corresponding to the occurrence of: (i) isolated water deficits at each of three growth stage, (ii) water deficit for two stages, (iii) water deficit for the all three growth stages studied. Water deficit applied was equivalent to half of the water needs of the crop. Results indicated that the sensibility coefficients decreased in the following order, for the different growing stages of the crop: initial tuber formation, tuber development and vegetative stage (when the three stages were considered separately). For conditions of deficit in two stages, values of $\mathrm{k}_{\mathrm{y}}$ were above 1, reaching the highest value for the deficit situation corresponding to initial tuber formation and tuber development.
\end{abstract}

Key words: Solanum tuberosum, sensibility coefficient, growth stages

\footnotetext{
${ }^{1}$ Professor Doutor do Departamento de Engenharia Agrícola, Centro de Ciências Agrárias, UFC, CP 12.168, CEP 60450 - 760, Fortaleza, CE, Fone: (085) 288 9754, Fax: (085) 2889755

${ }^{2}$ Professor Doutor, Departamento de Física e Meteorologia, Escola Superior de Agricultura "Luiz de Queiroz" - USP. CP 9, CEP 13418 - 900, Piracicaba, SP, Bolsista do CNPq

${ }^{3}$ Prof. Titular, Ph.D., Departamento de Horticultura, Escola Superior de Agricultura "Luiz de Queiroz" - USP. CP 9, CEP 13418 - 900, Piracicaba, SP
} 


\section{INTRODUÇÃO}

A escassez e o preço da água têm gerado preocupações que justificam estudos das relações entre déficit hídrico e produtividade; assim, a sistematização da freqüência de irrigação através do coeficiente sensibilidade da cultura $\left(\mathrm{k}_{\mathrm{y}}\right)$ proposto por Doorenbos \& Kassam (1979) permite planejar melhor a quantificação e o uso da água pela cultura, a fim de que se tenha uma irrigação mais eficiente. Para esses autores, $\mathrm{k}_{\mathrm{y}}$ é definido como uma relação empírica entre a queda de rendimento relativo, expressa por $\left(1-y_{a} / y_{m}\right)$, em que $y_{a}$ e $y_{m}$ são os rendimentos da cultura real e máximo obtidos e o déficit de evapotranspiração relativa, expresso por $\left(1-\mathrm{ET}_{\mathrm{a}} / \mathrm{ET}_{\mathrm{m}}\right)$ sendo $\mathrm{ET}_{\mathrm{a}}$ e $\mathrm{ET}_{\mathrm{m}}$ as evapotranspirações real e máxima, respectivamente. Com esta relação é possível se planejar, projetar e operar sistemas de irrigação, levando-se em conta o efeito de diferentes condições de manejo de água sobre a produção das culturas.

A FAO apresenta, no Boletim 33, os valores de $\mathrm{k}_{\mathrm{y}}$ de 26 culturas irrigadas, obtidos através da avaliação de inúmeros resultados de pesquisa encontrados na literatura, abrangendo ampla faixa de condições de crescimento nos estádios fenológicos. Para a cultura da batata, os valores desse coeficiente foram de $0,45,0,8,0,7$ e 0,2 nos estádios vegetativo, estolonização mais início da tuberização, formação da colheita e maturação, respectivamente; para o período vegetativo total da cultura, $\mathrm{k}_{\mathrm{y}}$ foi estimado em 1,1 (Doorenbos \& Kassam, 1979).

Shalhevet et al. (1983) apresentaram, para a cultivar Desire nas condições áridas de Negev, em Israel, as seguintes equações da função de produção na forma do modelo de Stewart \& Hagan, em cultura irrigada por dois sistemas de irrigação, indicando o efeito da forma de aplicação de água sobre o rendimento relativo: Aspersão:

$$
\mathrm{y}_{\mathrm{a}} / \mathrm{y}_{\mathrm{m}}=-0,134+1,15 \mathrm{ET}_{\mathrm{a}} / \mathrm{ET}_{\mathrm{m}}, \quad \mathrm{r}=0,982
$$

Gotejamento:

$$
\mathrm{y}_{\mathrm{a}} / \mathrm{y}_{\mathrm{m}}=-0,007+1,04 \mathrm{ET}_{\mathrm{a}} / \mathrm{ET}_{\mathrm{m}}, \quad \mathrm{r}=0,976
$$

Larsen (1982) aplicou o modelo sugerido por Doorenbos \& Kassam (1979) para determinar o fator de sensibilidade $\left(\mathrm{k}_{\mathrm{y}}\right)$ para os estádios vegetativo, início de tuberização e enchimento dos tubérculos da cultivar Russet Burbank, sujeita a déficit hídrico nesses estádios. Os valores do $\mathrm{k}_{\mathrm{y}}$ nos três estádios estudados para produção total foram de $0,4,1,33$ e 0,2 , respectivamente.

Stark \& McCann (1992) usaram o modelo de Stewart et al. (1983) para estimar o fator de sensibilidade $\left(\mathrm{k}_{\mathrm{y}}\right)$ para a cultivar de batata Russet Burbank, a partir de dados experimentais obtidos em dois anos, envolvendo dois níveis de déficits hídricos, distribuídos no início (I), no meio (M) e no final (F) do estádio enchimento dos tubérculos. Os maiores valores de $\mathrm{k}_{\mathrm{y}}$ foram encontrados para o rendimento de tubérculos da classe US $\mathrm{n}^{\circ} 1$, variando de 1,07 a 1,47; para o rendimento total, ky variou de 0,69 a 1,0 e o valor do ky igual a 1,47 ocorreu na seqüência de déficit aplicado no início e no meio do estádio de enchimento de tubérculos e ky $=1,0$ na seqüência de déficit no meio e no final do estádio estudado, ambos no estresse de $20 \%$ de ET.

Este trabalho tem como objetivo principal determinar o coeficiente de sensibilidade da batata $\left(\mathrm{k}_{\mathrm{y}}\right)$, cultivar "Monalisa", ao déficit hídrico nos estádios isolados e no ciclo total da cultura, usando-se o modelo sugerido por Doorenbos \& Kassam (1979).

\section{MATERIAL E MÉTODOS}

O trabalho foi conduzido na área experimental da Escola Superior de Agricultura Luiz de Queiroz - USP, Departamento de Horticultura, em Piracicaba, SP. O solo é classificado como Latossolo roxo, bem drenado, série Luiz de Queiroz (Ranzani et al., 1966). As características físicas e químicas do solo da área experimental estão apresentadas nas Tabelas 1 e 2.

Tabela 1. Características físicas do solo

\begin{tabular}{lccc}
\hline \multicolumn{1}{c}{ Características } & \multicolumn{3}{c}{ Camada (m) } \\
\cline { 2 - 4 } & $0-0,15$ & $0,15-0,30$ & $0,30-0,45$ \\
\hline Granulometria (\%) & 37,64 & 23,52 & 22,08 \\
Areia & 32,84 & 14,14 & 16,98 \\
Silte & 29,53 & 62,34 & 60,94 \\
Argila & Barrento & Argiloso & Argiloso \\
Classificação textural & 1.365 & 1.526 & 1.425 \\
Densidade do solo $\left(\mathrm{kg} \mathrm{m}^{-3}\right)$ & & & \\
\hline
\end{tabular}

Tabela 2. Características químicas do solo da área experimental

\begin{tabular}{|c|c|c|c|c|c|c|c|c|c|}
\hline$\overline{\mathrm{pH}}$ & Pres. & M.O. & $\mathrm{Ca}^{2+}$ & $\mathrm{Mg}^{2+}$ & $\mathrm{K}^{+}$ & $\mathrm{H}^{+}+\mathrm{Al}^{3+}$ & CTC & S & V \\
\hline $\mathrm{CaCl}_{2}$ & $\mathrm{Mg} \mathrm{dm}^{-3}$ & $\mathrm{~g} \mathrm{dm}^{-3}$ & - & 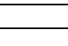 & $\mathrm{cn}$ & $\mathrm{l}_{\mathrm{c}} \mathrm{dm}^{-3}$ & & & $\%$ \\
\hline 5,75 & 460,50 & 26,70 & 4,41 & 1,30 & 0,65 & 2,71 & 9,07 & 6,36 & 70,0 \\
\hline
\end{tabular}
na camada de $0-0,20 \mathrm{~m}$

Os dados experimentais relativos à produtividade de tubérculos foram obtidos do ensaio de campo com a cultivar Monalisa, em uma área cultivada de $1350 \mathrm{~m}^{2}$, sendo que as parcelas experimentais foram instaladas em duas áreas contíguas de $42 \mathrm{~m}$ de comprimento e 4,6 m de largura cada uma, sob abrigo com cobertura móvel de plástico transparente de polietileno de 200 micra de espessura, que foram usados para proteger as parcelas, quando chovia. $\mathrm{O}$ delineamento experimental adotado foi blocos ao acaso, com oito tratamentos e quatro repetições. A unidade experimental foi constituída por cinco linhas de $2,1 \mathrm{~m}$ de comprimento, espaçadas $0,8 \mathrm{~m}$. Cada parcela ocupou uma área de $8,4 \mathrm{~m}^{2}$, com total de 30 plantas por parcela. A área útil da parcela foi constituída pelas três linhas centrais, onde foram feitas as avaliações de produtividade e umidade do solo. Os tratamentos foram definidos em função da época de indução do déficit hídrico nos estádios vegetativo, de tuberização e de enchimento de tubérculos. Aplicaram-se os seguintes tratamentos: (1) testemunha, sem déficit hídrico; (2) com déficit hídrico no estádio enchimento de tubérculos; (3) com déficit no estádio de tuberização; (4) com déficit nos estádios tuberização e enchimento de tubérculos; (5) com déficit no estádio vegetativo; (6) com déficit nos estádios vegetativo e enchimento de tubérculos; (7) com déficit nos estádios vegetativo e tuberização e (8) nos três estádios fenológicos. Os estádios fenológicos foram definidos de acordo com o critério adotado por Kleinkopf (1983) para a batata e a identificação do início dos estádios de tuberização e enchimento de tubérculos foi feito por inspeções em plantas cultivadas em volta das parcelas experimentais, a partir dos vigésimo e quadragésimo dias após a emergência da cultura, respectivamente. 
O déficit hídrico correspondeu à aplicação da metade da lâmina média d'água aplicada ao tratamento sem déficit hídrico, calculada a partir do potencial matricial da água no solo, nas profundidades de 10 e $30 \mathrm{~cm}$; assim, nas parcelas sem déficit hídrico e quando o potencial matricial de água no solo atingia $20 \mathrm{kPa}$, irrigava-se aplicando-se uma lâmina que levasse o solo à condição de capacidade de campo, pois o potencial da água no solo favorável à produção máxima da batata situa-se acima de -30 kPa (Singh et al., 1968; Yadav \& Tripathi, 1973). Os valores da umidade no solo foram obtidos, no momento das irrigações, das curvas características de água no solo, nas profundidades de 10 e $30 \mathrm{~cm}$, a partir dos valores de potencial da água.

As lâminas de irrigação aplicadas nas parcelas sem déficit hídrico foram definidas em função do potencial da água do solo nas profundidades de 0,10 e $0,30 \mathrm{~m}$. As expressões para o cálculo das lâminas de irrigação são mostradas a seguir:

Camada $(0-20) \mathrm{cm}$

$$
\mathrm{L}_{(0-20)}=\left(q_{c c 10}-q_{a 10}\right) \cdot 200
$$

Camada $(20-40) \mathrm{cm}$

$$
L_{(20-40)}=\left(q_{c c 30}-q_{a 30}\right) .200
$$

em que:

$\mathrm{L}_{(0-20)}$ e $\mathrm{L}_{(20-40)}$ - lâmina de irrigação para as camadas de $0 \mathrm{a}$ $20 \mathrm{~cm}$ e de 20 a $40 \mathrm{~cm}$ respectivamente $(\mathrm{mm})$

$q_{c c 0,10}$ e $q_{c c 0,30}$ - umidade do solo correspondente às capacidades de campo, nas profundidades de $10 \mathrm{e}$ $30 \mathrm{~cm}$, expressa em $\mathrm{cm}^{3} \mathrm{~cm}^{-3}$, respectivamente

$q_{a 10}$ e $q_{a 30}$ - umidade do solo no momento das irrigações, nas profundidades de 10 e $30 \mathrm{~cm}$, respectivamente, em $\mathrm{cm}^{3} \mathrm{~cm}^{-3}$

As evapotranspirações real e máxima da cultura foram estimadas a partir do balanço hídrico, que tem seu fundamento na lei da conservação das massas (Reichardt, 1985). Considerouse um volume de solo de $40 \mathrm{~cm}$ para as determinações de todos os componentes do balanço hídrico da cultura. Esta profundidade abrange o máximo do sistema radicular da batata (Lesczynski \& Tanner, 1976; Swaminathan \& Verma, 1977; Rab \& Willatt, 1987). Foi usada, em cada parcela, uma bateria de tensiômetros com manômetros de mercúrio nas profundidades de $10,30,50$ e $70 \mathrm{~cm}$ para medidas dos potenciais matriciais da água no solo. As leituras foram feitas diariamente, entre 7 e 8 horas da manhã e, nas condições em que se conduziu o trabalho, os componentes precipitação e escoamento superficial do balanço hídrico foram desprezados, uma vez que as parcelas foram protegidas contra as chuvas e, também, sistematizadas e com bordos para evitar o escoamento superficial da água de irrigação. O cálculo do balanço hídrico teve início a partir de 15 dias após a emergência, para que a fase de estabelecimento da cultura não apresentasse problemas e foi realizado nos três estádios fenológicos em estudo.

Para o cálculo do fator sensibilidade das culturas ao déficit hídrico, utilizou-se a seguinte expressão empírica descrita por Doorenbos \& Kassam (1979):

$$
\mathrm{k}_{\mathrm{y}}=\frac{1-\left(\mathrm{y}_{\mathrm{a}} / \mathrm{y}_{\mathrm{m}}\right)}{1-\left(\mathrm{ET}_{\mathrm{a}} / \mathrm{ET}_{\mathrm{m}}\right)}
$$

em que:

$\mathrm{k}_{\mathrm{y}}$ - fator de sensibilidade parcial para cada estádio fenológico

$\mathrm{y}_{\mathrm{a}}$ - rendimento real da cultura obtido no tratamento submetido a déficit hídrico $\left(\mathrm{Mg} \mathrm{ha}^{-1}\right)$

$\mathrm{y}_{\mathrm{m}}$ - rendimento máximo da cultura obtido no tratamento, que não sofreu déficit hídrico $\left(\mathrm{Mg} \mathrm{ha}^{-1}\right)$

$\mathrm{ET}_{\mathrm{a}}$ - evapotranspiração real da cultura, obtida nos tratamentos submetidos a déficit hídrico $\left(\mathrm{mm} \mathrm{d}^{-1}\right)$

$\mathrm{ET}_{\mathrm{m}}$ - evapotranspiração máxima da cultura obtida no tratamento sem déficit $\left(\mathrm{mm} \mathrm{d}^{-1}\right)$

A semeadura foi realizada no dia 27 de agosto de 1993, em sulcos distanciados $0,8 \mathrm{~m}$; as batatas-semente foram distribuídas em sulcos de aproximadamente $0,07 \mathrm{~m}$ de profundidade, manualmente, no espaçamento de $0,35 \mathrm{~m}$, juntamente com o inseticida Aldicarbe e, em seguida, cobertas por solo de um sulco aberto ao lado, onde foram distribuídos os adubos e posteriormente misturados à terra, com um sulcador manual. A adubação de semeadura, comum a todas as parcelas, correspondeu a $60 \mathrm{~kg} \mathrm{ha}^{-1} \mathrm{de} \mathrm{N}$, a $240 \mathrm{~kg} \mathrm{ha}^{-1}$ de $\mathrm{P}_{2} \mathrm{O}_{5}$ e $120 \mathrm{~kg} \mathrm{ha}^{-1}$ de $\mathrm{K}_{2} \mathrm{O}$, segundo recomendação sugerida por Freire et al. (1981) para solos com teores médios e altos de $\mathrm{P}$ e K, respectivamente. As doses de $\mathrm{P}_{2} \mathrm{O}_{5}$ e $\mathrm{K}_{2} \mathrm{O}$ foram aplicadas na semeadura, e a de $\mathrm{N}$ foi parcelada, sendo metade aplicada na ocasião da semeadura e a outra 35 dias após a emergência, durante a operação de amontoa.

As parcelas foram irrigadas da semeadura até o início da aplicação dos tratamentos, por um sistema convencional de irrigação por aspersão. Neste período, foi aplicado uma lâmina média total de $65,9 \mathrm{~mm}$, com freqüência média de 5 dias. Aos quinze dias após a emergência verificou-se a uniformidade do estande, seguida então, da aplicação dos tratamentos, sendo a água aplicada por um equipamento em forma de T, feito de PVC rígido de $1 / 2$ polegada de diâmetro, com furos de $1 / 16$ polegada para sair a água, e distanciados de $2 \mathrm{em} 2 \mathrm{~cm}$, tendo um hidrômetro de vazão máxima $1,5 \mathrm{~m}^{3} \mathrm{~h}^{-1}$ e resolução de $0,0001 \mathrm{~m}^{3}$ para melhor controle do volume de água aplicado às parcelas.

Durante o ciclo da cultura foram realizadas duas capinas manuais com amontoa para favorecer o desenvolvimento das plantas. Para controle das pragas e doenças foram feitas aplicações semanais dos produtos Tamaron, Manzate, Dithane 45 e Mancozeb. Nas colheitas, realizadas manualmente aos 81 dias após emergência, os tubérculos colhidos da área útil foram pesados e classificados de acordo com o diâmetro transversal, como: especial: > 45 mm; média: de 33 a 45 mm; miúda: de 23 a 33 mm; e miudinha: de 20 a 23 mm, segundo Bittencourt et al.(1985).

\section{RESULTADOS E DISCUSSÃO}

\section{Déficit de evapotranspiração relativa ( 1 - ET $_{\mathrm{a}} / \mathbf{E T}_{\mathrm{m}}$ )}

Na Figura 1 são mostradas as variações dos déficits de evapotranspiração relativa para cada estádio de desenvolvimento da batata, obtidos a partir da média ponderada das quatro repetições, nos sete tratamentos que sofreram déficits hídricos. Nota-se claramente que os déficits aplicados em cada estádio não foram os mesmos entre os tratamentos e no estádio vegetativo, o déficit de evapotranspiração relativa variou entre 
17 e $25 \%$, cujo maior valor se deu no Tratamento 5, provocando redução na produtividade da batata de $14,2 \%$ em relação ao Tratamento 1, sem déficit (Tabela 1); no estádio seguinte, tuberização, o déficit de evapotranspiração relativa variou de 33 a $43,5 \%$.

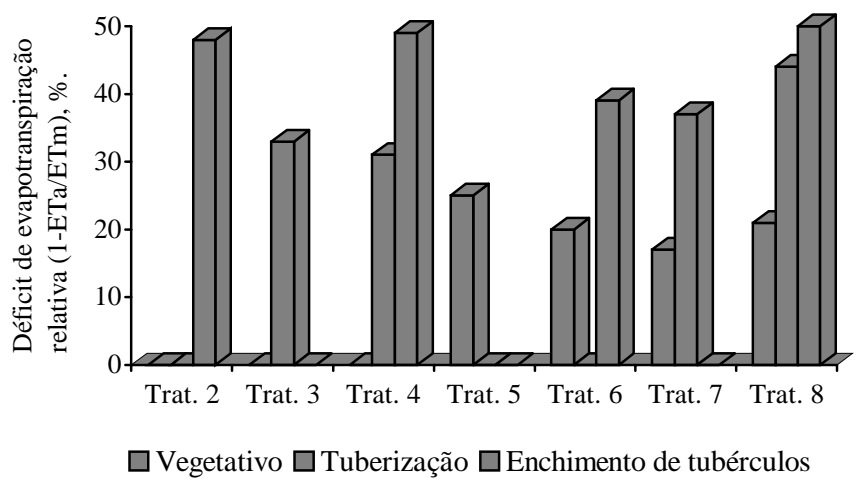

Figura 1. Déficit de evapotranspiração relativa (1-ETa/ETm) durante os estádios fenológicos da batata nos tratamentos sob déficit hídrico

O Tratamento 3, caracterizado por déficit hídrico apenas no estádio de tuberização, apresentou déficit de evapotranspiração relativa média de $33,4 \%$, causando redução na produção, de $60,1 \%$, em relação ao Tratamento 1 (Tabela 1), com o que se conclui que o completo suprimento de água nesse estádio é extremamente importante, não permitindo qualquer eliminação de irrigação. No estádio de enchimento de tubérculos o déficit variou de 39 a $49 \%$, havendo tendência de uniformidade de déficit entre tratamentos nesse estádio; apenas o Tratamento 6 sofreu déficit de aproximadamente $39,5 \%$, enquanto os demais tiveram déficit entre 47 a $49 \%$.

O déficit de evapotranspiração relativa de $47,5 \%$ no Tratamento 2 , resultou em uma redução na produção de tubérculos, de 51,46\% (Tabela 1). A aplicação do déficit neste estádio, aproximadamente $1 / 3$ maior que no estádio de tuberização, causou redução em escala menor do que quando o déficit foi aplicado no estádio de tuberização. Deduz-se, portanto, que a existência de qualquer déficit hídrico no estádio de enchimento de tubérculos pode ser prejudicial à produtividade geral, mas em menor escala que no estádio de tuberização.

O controle do nível de água no solo é difícil, podendo-se afirmar que a variação do déficit de evapotranspiração entre as diferentes fases foi grande, em conseqüência das diferenças de duração dos estádios e das características de cada tratamento.
Os resultados obtidos neste trabalho confirmaram as observações de Doorenbos \& Kassam (1979) e Jana et al. (1989) de que o efeito do déficit hídrico sobre a produção de batata varia com o estádio do ciclo fenológico da cultura e que as maiores reduções na produção se dão na fase de estolonização e início da tuberização. Quanto aos tratamentos submetidos a déficits em dois ou três estádios observa-se, na Figura 1, que o Tratamento 8 apresentou maior déficit de evapotranspiração relativa acumulada, o que resultou na redução do rendimento de 70,5\%, em média, em relação ao Tratamento 1 (Tabela 1); para os tratamentos que sofreram deficiência de água em dois estádios fenológicos, o Tratamento 4, com déficits de evapotranspiração relativa de $30,5 \%$ no estádio de tuberização e de $48,7 \%$ no estádio de enchimento de tubérculos, resultou em uma redução na produtividade, de $65,9 \%$, maior que nos outros tratamentos que também tiveram deficiência em dois estádios fenológicos, (Tabela 1); já os tratamentos 6 e 7, em que ambos tiveram primeiro déficit de evapotranspiração relativa no estádio vegetativo, a redução na produtividade foi de $48,16 \%$ no Tratamento 6 , que teve déficits de evapotranspiração relativa de $19,93 \%$ no estádio vegetativo e de $39,28 \%$ no estádio de enchimento de tubérculos, sendo este efeito maior que no Tratamento 7 , cujos déficits de evapotranspiração relativa foram de $16,95 \%$ no estádio vegetativo e de $37,39 \%$ no estádio tuberização, causando redução na produtividade, de 41,28\% (Tabela 1).

\section{Fator de sensibilidade ao déficit hídrico (ky)}

A Tabela 1 apresenta os dados médios de $\mathrm{ET}_{\mathrm{a}}$ e $\mathrm{ET}_{\mathrm{m}}, \mathrm{y}_{\mathrm{a}}, \mathrm{y}_{\mathrm{m}}$, $\left(1-\mathrm{ET}_{\mathrm{a}} / \mathrm{ET}_{\mathrm{m}}\right),\left(1-\mathrm{y}_{\mathrm{a}} / \mathrm{y}_{\mathrm{m}}\right)$ e os coeficientes de sensibilidade $\mathrm{k}_{\mathrm{y}}$ médios, calculados para cada tratamento. De acordo com Doorenbos \& Kassam (1979) quanto maior o valor de $\mathrm{k}_{\mathrm{y}}$, maiores as perdas no rendimento da cultura, em condições de déficit hídrico. Observando-se os valores médios de $\mathrm{k}_{\mathrm{y}}$ dos Tratamentos 2, 3 e 5, submetidos a déficit hídrico em apenas um estádio fenológico da cultura, constata-se que a redução relativa da produtividade da batata foi mais acentuada no estádio de tuberização (Tratamento 3 ) com valor de $\mathrm{k}_{\mathrm{y}}=1,53$. A intensidade desse efeito no Tratamento 2 , com déficit no estádio de enchimento de tubérculos, foi menor que no Tratamento 3, com $\mathrm{k}_{\mathrm{y}}=0,92$. O efeito do déficit hídrico sobre o rendimento foi menos acentuado no estádio vegetativo (Tratamento 5), $\operatorname{com~}_{\mathrm{y}}=0,57$, valor menor que nos outros estádios. O déficit hídrico no estádio vegetativo deu-se apenas a partir da segunda parte do estádio, não se podendo afirmar qual seria o efeito sobre o valor de $k_{\mathrm{y}} \mathrm{se}$ o déficit fosse aplicado a partir do início do estádio vegetativo.

Tabela 1. Valores de $\left(1-\mathrm{ET}_{\mathrm{a}} / \mathrm{ET}_{\mathrm{m}}\right),\left(1-\mathrm{y}_{\mathrm{a}} / \mathrm{y}_{\mathrm{m}}\right)$ e do fator de sensibilidade, ky, do cultivar "Monalisa" nos tratamentos sob déficit hídrico

\begin{tabular}{crrcrcrc}
\hline Tratamento & \multicolumn{1}{c}{$\mathrm{ET}_{\mathrm{a}}{ }^{1}$} & \multicolumn{1}{c}{$\mathrm{ET}_{\mathrm{m}}{ }^{2}$} & $\left(1-\mathrm{ET}_{\mathrm{a}} / \mathrm{ET}_{\mathrm{m}}\right)$ & \multicolumn{1}{c}{$\mathrm{y}_{\mathrm{a}}{ }^{3}$} & $\mathrm{y}_{\mathrm{m}}{ }^{3}$ & $\left(1-\mathrm{y}_{\mathrm{a}} / \mathrm{y}_{\mathrm{m}}\right)$ & $\mathrm{k}_{\mathrm{y}}$ \\
\hline 2 & 56,09 & 119,74 & 0,532 & 11,04 & 21,51 & 0,487 & 0,92 \\
3 & 41,03 & 67,52 & 0,392 & 8,63 & 21,51 & 0,599 & 1,53 \\
4 & 100,13 & 187,26 & 0,465 & 7,44 & 21,51 & 0,654 & 1,41 \\
5 & 34,25 & 45,62 & 0,249 & 18,45 & 21,51 & 0,142 & 0,57 \\
6 & 94,98 & 165,37 & 0,426 & 10,44 & 21,51 & 0,515 & 1,21 \\
7 & 77,71 & 113,14 & 0,313 & 12,63 & 21,51 & 0,413 & 1,32 \\
8 & 123,87 & 232,88 & 0,468 & 6,34 & 21,51 & 0,705 & 1,51 \\
\hline
\end{tabular}

Valores da evapotranspiração real acumulada no período de déficit hídrico $(\mathrm{mm})$

Valores da evapotranspiração máxima acumulada no tratamento 1, no mesmo período da ETa acumulada nos tratamentos sob déficit hídrico (mm)

3 Produtividade real $\left(\mathrm{y}_{\mathrm{a}}\right)$ e máxima $\left(\mathrm{y}_{\mathrm{m}}\right)\left(\mathrm{Mg} \mathrm{ha}^{-1}\right)$ 
A variação do fator de sensibilidade ao déficit hídrico ao longo dos estádios fenológicos estudados, é a mesma encontrada no trabalho de Doorenbos \& Kassam (1979) mas o valor para o estádio de tuberização é bastante diferente $\left(\mathrm{k}_{\mathrm{y}}=0,8\right.$ para a FAO, contra $\mathrm{k}_{\mathrm{y}}=1,5$ para o presente estudo). O mesmo acontece com os estádios de enchimento de tubérculos $\left(\mathrm{k}_{\mathrm{y}}=0,7\right.$ para a FAO, contra $\mathrm{k}_{\mathrm{y}}=0,9$ para o presente estudo) e vegetativo $\left(\mathrm{k}_{\mathrm{y}}=0,45\right.$ para a FAO, contra $\left.\mathrm{k}_{\mathrm{y}}=0,6\right)$ mas com discrepância menor. Considera-se que um $\mathrm{k}_{\mathrm{y}}$ inferior a 1 significa baixa sensibilidade da cultura ao déficit hídrico, o que parece não acontecer com a batata.

Nos tratamentos com déficit hídrico em mais de um estádio fenológico, os valores de $\mathrm{k}_{\mathrm{y}}$ foram maiores que 1 , em todos os tratamentos. O Tratamento 8, que foi submetido a déficit hídrico nos três estádios fenológicos, apresentou o maior valor de $\mathrm{k}_{\mathrm{y}} \mathrm{e}$ nos tratamentos com déficit em dois estádios, o maior valor de $\mathrm{k}_{\mathrm{y}}$ deu-se no Tratamento 4, caracterizado por déficit hídrico nos estádios de tuberização e enchimento de tubérculos, o que era esperado, tendo em vista que o peso do déficit hídrico nesses estádios é maior que nos estádios vegetativo e de tuberização, ou vegetativo e enchimento de tubérculos. $\mathrm{O}$ valor de $\mathrm{k}_{\mathrm{y}}$ no Tratamento 4 é aproximadamente igual ao produto entre os valores dos Tratamentos 2 e 3 , que sofreram déficit hídrico nos estádios de enchimento de tubérculos e tuberização, respectivamente. A tendência de diminuição dos valores de $\mathrm{k}_{\mathrm{y}}$, quando se tem déficit em dois ou três estádios, é justificável quando se tem valores de $\mathrm{k}_{\mathrm{y}}$ menores que $1 \mathrm{em}$ um ou dois estádios, como é o caso do presente estudo, se considerado um efeito multiplicativo; entretanto, nos casos dos Tratamentos 6,7 e 8 , a multiplicação dos valores de $\mathrm{k}_{\mathrm{y}}$ obtidos nos tratamentos em que o déficit foi imposto em cada estádio isoladamente, não conduz ao valor igual ao obtido para os tratamentos com déficit em mais de um estádio. Esta igualdade não foi verificada entre o valor de $\mathrm{k}_{\mathrm{y}}$ do Tratamento 7 confrontado com a multiplicação dos valores de $\mathrm{k}_{\mathrm{y}}$ para os Tratamentos 3 e 5 , nem com o Tratamento 6 com os Tratamentos 2 e 5 e o Tratamento 8 com os Tratamentos 2,3 e 5 , sendo os produtos sempre menores que os valores de $\mathrm{k}_{\mathrm{y}}$ encontrados nos Tratamentos 6, $7 \mathrm{e} 8$. A literatura mostra que não há consenso entre os autores de como os efeitos do déficit hídrico em cada estádio fenológico se traduzem num efeito geral sobre a produtividade, isto é, se seria um efeito multiplicativo, fato não constatado em alguns dos tratamentos aqui estudados, aditivo ou de outra natureza (Stewart et al., 1975; Vaux et al., 1981).

\section{CONCLUSÕES}

1. Os valores do $\mathrm{k}_{\mathrm{y}}$ obtidos no presente experimento foram superiores aos valores apresentados na bibliografia, o que ressalta a necessidade do cálculo desta relação para o local e a cultivar escolhida.

2. Para condições de déficits hídricos em apenas um estádio fenológico da cultura da batata, os valores de ky estimados pelo modelo sugerido por Doorenbos e Kassam (1979) variaram na seguinte ordem decrescente: tuberização, enchimento de tubérculos e vegetativo.

3. Para condições de déficits em dois estádios, os valores de $\mathrm{k}_{\mathrm{y}}$ foram todos superiores a 1 , tendo alcançado o maior valor na situação de déficits nos estádios tuberização e enchimento de tubérculos.

\section{REFERÊNCIAS BIBLIOGRÁFICAS}

BITTENCOURT, C.; REIFSCHINEIDER, F. J. B.; MAGALHÃES, J. R. de; FURUMOTO, O.; FEDALTO, A. A.; MARQUELLI. W. A.; SILVA, H. R. de; FRANÇA, F. H.; AVILA, A. C. de; GIORDANO, L. B. de. Cultivo da batata (Solanum tuberosum L.). Brasília, EMBRAPA/CNPH, 1985. 20p. Instruções Técnicas do CNPHortaliças, 8 .

DOORENBOS, J.; KASSAM, A.H. Efectos del agua sobre el rendimiento de los cultivos. Roma: FAO, 1979. 212p, FAO. Riego Drenage, 33.

FREIRE, F. M.; MARTINS FILHO, C.A.S.; MONNERAT, P.H. Nutrição mineral e adubação da batata. Informe Agropecuário, Belo Horizonte, v. 7, n.76, p. 24-30, 1981.

JANA, P.K. das, S.; MANDAL, B. B.; BANDYOPADHYAY, P. Effect of soil moisture tension at different physiological stages of growth on yield and consumptive use of water by potato. Environment and Ecology, New Delhi, v. 7, n.4, p. 809-812, 1989.

KLEINKOPF, G. E. Potato. In: TEARE, I.D. ; PEET, M.M. Crop - water relations. New York: Wiley Internscience, 1983. p.285-305.

LARSEN, D. C. Simplifying potato irrigation scheduling the Idaho program. American Potato Journal, Orono, v.61, n.4, p.215-27, 1982

LESCZYNSKI, D. B.; TANNER, C. B. Seasonal variation of root distribution of irrigated, field grown Russet Burbank potato. American Potato Journal, Orono, v.53, n.2, p.69-78, 1976.

RAB, M. A.; WILLATT, S. T. Water use by irrigated potatoes on a duplex soil. Australian Journal of Experimental Agriculture, East Melbourne, v. 27, n.1, p.165-72, 1987.

RANZANI, G. ; FREIRE, O. ; KINJO, T. Carta de solos do município de Piracicaba. Piracicaba: Centro de Estudos de Solos - ESALQ/USP, 1966. 85p.

REICHARDT, K. Processo de transferência no sistema soloplanta-atmosfera. 4. ed. Campinas: Fundação Cargill, 1985. 466p.

SHALHEVET, J. ; SHIMSHI, D. ; MEIR, T. Potato irrigation requirements in a hot climate using sprinkler and drip methods. Agronomy Journal, Madison, v.75, n.1, p.13-6, 1983.

SINGH, M. ; HUKKERI, S. B. ; SINGH, N. B. Response of potato to varying moisture regimes, nitrogen, phosphate and potassium. Indian Journal of Agricultural Sciences, New Delhi, v.38, n.1, p.76-89, 1968.

STARK, J. C. ; McCANN, I. R. Optimal allocation of limited water supplies for Russet Burbank potatoes. American Potato Journal, Orono, v.69, n.7, p.413-421, 1992.

STEWART, J. I. ; MISRA, R. D. ; PRUITT, W. O.; HAGAN, R. M. Irrigating corn and grain sorghum with a deficient water supply. Transactions of the ASAE, St. Joseph, v.18, n.2, p.270-280, 1975.

SWAMINATHAN, K. ; VERMA, B. C. Active root distribution profiles of Indian potato cultivars in relation to extraction of phosphorus from the soil. Journal of Nuclear Agriculture and Biology, New Delhi, v.6, p.26-28, 1977.

VAUX Jr., H. J. ; PRUITT, W. O. ; HATCHETT, S. A.; SOUSA, F. de. Optimization of water use with respect to crop production. Davis, California: Department of Water Resources, 1981. 174p., Technical Completion Report, 13 - 53395.

YADAV, S. C.; TRIPATHI, B. R. Water requirement of potato. Indian Journal of Agricultural, New Delhi, v.43, n.5, p.477-482, 1973. 